\title{
Sequential reconstruction for recurrent head and neck cancer: A 10-year experience
}

\author{
Soon Won Chung, Il Hwan Byun, Won Jai Lee \\ Department of Plastic and Reconstructive Surgery, Institute for Human Tissue Restoration, Yonsei University College of Medicine, Seoul, \\ Korea
}

Background Most patients with head and neck cancer successfully undergo oncologic resection followed by free or local flap reconstruction, depending on the tumor's size and location. Despite effective curative resection and reconstruction, head and neck cancer patients still face a high risk of recurrence and the possibility of a second primary cancer. Moreover, surgeons hesitate to perform sequential reconstruction following curative resection for several reasons. Few large-scale studies on this subject are available. Therefore, we retrospectively evaluated the outcome of sequential head and neck reconstruction to determine the possible risks.

Methods In total, 467 patients underwent head and neck reconstruction following cancer resection at our center from 2008 to 2017. Of these cases, we retrospectively reviewed the demographic and clinical features of 58 who had sequential head and neck reconstruction following resection of recurrent cancer.

Results Our study included 43 males (74.1\%) and 15 females (25.9\%). The mean age at the initial operation was $55.4 \pm 15.3$ years, while the mean age at the most recent operation was $59.0 \pm 14.3$ years. The interval between the first and second operations was $49.2 \pm 62.4$ months. Twelve patients (20.7\%) underwent surgery on the tongue, and $12(20.7 \%)$ had procedures on the oropharynx. Thirty-four patients $(58.6 \%)$ received a sequential free flap reconstruction, and 24 patients (41.4\%) were treated using locoregional flaps. No cases of flap failure occurred.

Conclusions Our findings suggest that patients who need additional operations with recurrent head and neck cancer could optimally benefit from sequential curative resections and reconstructions.

Keywords Head and neck neoplasms / Microsurgery
Correspondence: Won Jai Lee Department of Plastic and Reconstructive Surgery, Institute for Human Tissue Restoration, Yonsei University College of Medicine, 50 Yonsei-ro, Seodaemun-gu, Seoul 03722, Korea

Tel: $+82-2-2228-2218$

Fax: +82-2-577-4914

E-mail: pswjlee@yuhs.ac

Received: January 25, $2019 \bullet$ Revised: July 17, $2019 \bullet$ Accepted: July 26, 2019

pISSN: 2234-6163 • elSSN: 2234-6171 • https://doi.org/10.5999/aps.2019.00157• Arch Plast Surg 2019;46:449-454

\section{INTRODUCTION}

Most patients with head and neck cancer successfully receive oncologic resection followed by free or local flap reconstruction, depending on the tumor's size and location [1,2]. Locoregional flaps, such as pectoralis major muscle island flaps or temporalis muscle flap transpositions, are widely used. In addition to local flaps, microvascular free flap surgery - with options including anterolateral thigh free flaps, radial forearm free flaps, and vertical rectus abdominis myocutaneous free flaps-is also em- 
ployed because this technique can yield both cosmetic and functional reconstruction in one step [3]. With a flap failure rate of less than 5\% at most institutions, surgical resection of the tumor followed by reconstruction can be performed without an undue burden [4]. Despite effective curative resection and reconstruction, head and neck cancer patients face a high risk of recurrence $[5,6]$. In addition, patients with a history of head and neck cancer are at risk of a second primary cancer at a rate of $5 \%-30 \%$ [7]. In these cases, surgeons inevitably choose additional treatments including operations. In these patients, the optimal treatment choice is considered to be sequential curative resection and reconstruction, just as in those with primary cancer [5]. Sequential reconstruction is defined as reconstruction following the resection of a recurrent tumor even though a previous reconstructive procedure was performed at the same site. However, due to a lack of recipient vessels, altered anatomy, risk of flap loss, and other complications, reasonable hesitation to perform sequential reconstructions often occurs before an additional operation $[4,8,9]$. Although these factors might negatively affect a secondary operation, few large-scale studies have investigated the operative outcomes of sequential reconstruction following recurrent head and neck cancer. Therefore, we conducted a retrospective review of sequential reconstructions performed during a 10-year period at our institution. We sought to determine whether additional microvascular or locoregional reconstruction was the optimal treatment strategy. In this study, we evaluated the outcomes of sequential reconstructions for patients who experienced recurrent head and neck cancer and postoperative complications associated with reoperation.

\section{METHODS}

A review of patients who experienced recurrent head and neck cancer followed by reconstruction with a locoregional flap or a free vascularized flap at our center between 2008 and 2017 was performed. Patients were included in our sample if they underwent reconstruction following curative resection of recurrent head and neck cancer, regardless of the reconstruction method used during the first procedure. Additional reconstructions due to nonmalignant lesions were not included in our sample. Thus, only patients who underwent sequential curative resection of recurrent head and neck cancer followed by reconstruction were included in this study.

Our review included 58 total patients who received sequential head and neck reconstructions. A retrospective review of their demographic characteristics and outcomes was carried out. We analyzed the cancer sites, amount of time until recurrence, pathology, the initial and recurrent cancer stages, and any chemo- therapy and radiotherapy that took place. The final reconstruction strategies were categorized as either a free flap or a local flap. In cases where a free vascularized flap was performed, micro-anastomosed arteries and veins were reconstituted. We also analyzed any postoperative complications. Continuous data are represented as mean \pm standard deviation.

\section{RESULTS}

A total of 457 patients underwent head and neck reconstruction following tumor resection. Among them, 58 patients underwent reconstruction following curative resection of recurrent head and neck cancer, including 43 males (74.1\%) and 15 females (25.9\%). The average follow-up period was $44.2 \pm 39.4$ months, with a range from 3 months to 143 months. Twenty-nine patients (50\%) died during follow-up due to old age, other medical conditions, and tumor recurrence. The 1-year survival rate was $74.1 \%$ and the 5 -year survival rate was $50 \%$. The number of patients who died after being diagnosed with a local recurrent tumor or distant metastasis was 15 (25.9\%). Fourteen patients (24.1\%) died for other reasons, including old age, heart, lung disease, and intracranial hemorrhage. There were no cases of failed reconstruction following curative resection of recurrent head and neck cancer at our institution. Patients' demographic characteristics, including tumor location, pathology, and stage, are listed in Table 1. The mean age of patients at the time of the initial operation was $55.4 \pm 15.3$ years, and the average age at the most recent procedure was $59.0 \pm 14.3$ years. The mean interval between operations was $49.2 \pm 62.4$ months. Twelve patients (20.7\%) underwent surgery on the tongue, 12 patients (20.7\%) on the oropharynx, nine patients (15.5\%) on the oral cavity, and seven patients (12.1\%) on the maxilla. The most common type of malignancy was squamous cell carcinoma, which was found in 43 patients (74.1\%), followed by adenocarcinoma in eight patients (13.8\%). At the time of the first operation, 19 patients (32.8\%) had stage I malignancy, while 15 (25.9\%) were diagnosed with stage IV malignancy. Upon recurrence, the largest number of patients had stage IV malignancy (24 patients; $41.4 \%$ ), followed by stage I malignancy (15 patients; $25.9 \%$ ). Twelve patients (22.4\%) underwent chemotherapy, while 29 (50.0\%) received radiotherapy. Twelve patients (20.7\%) experienced recurrence after sequential reconstruction, of whom two patients (3.4\%) underwent local excision and nine patients (15.5\%) died. Eight patients (13.8\%) were diagnosed with distant metastasis, with metastatic sites including the bone, lung, brain and neck, of whom six patients (10.3\%) died. Twenty patients (34.5\%) underwent postoperative radiotherapy and 18 patients (31.0\%) underwent postoperative chemotherapy after 


\section{Table 1. Patient characteristics}

\begin{tabular}{|lc|}
\hline Characteristics & Value \\
\hline No. of patients & 58 \\
Sex & \\
Male & $43(74.13)$ \\
Female & $15(25.86)$ \\
Age at initial operation (yr) & $55.40 \pm 15.27$ \\
Age at sequential operation (yr) & $59.04 \pm 14.29$ \\
Term between operations (mon) & $49.2 \pm 62.4$ \\
Follow-up period (mon) & $44.2 \pm 39.4$ \\
Reconstruction sites & \\
Tongue & $12(20.69)$ \\
Oropharynx & $12(20.69)$ \\
Oral cavity & $9(15.52)$ \\
Maxilla & $7(12.07)$ \\
Hypopharynx & $5(8.62)$ \\
Parotid gland & $5(8.62)$ \\
External auditory canal & $3(5.17)$ \\
Skin & $2(3.45)$ \\
Lacrimal gland & $2(3.45)$ \\
Mandible & $1(1.72)$ \\
Pathology & \\
Squamous cell carcinoma & \\
Adenocarcinoma & $43(74.13)$ \\
Mucoepidermoid carcinoma & $8(13.79)$ \\
Pleomorphic carcinoma & $2(3.45)$ \\
Malignant melanoma & $2(3.45)$ \\
Basal cell carcinoma & $10(17.24)$ \\
Myoepithelial carcinoma & \\
Initial stage & $13(1.72)$ \\
I & $1(1.72 .34)$ \\
II & $24(41.38)$ \\
III & $12(20.69)$ \\
IV & $29(50.00)$ \\
Recurrent stage & $1(1.72)$ \\
I & \\
II & $19(32.76)$ \\
Radiotherapy & $11(18.97)$ \\
$\geq 2$ Free flaps & $13(22.41)$ \\
\hline Values are presented as number (\%) or mean \pm SD. & $15(25.86)$ \\
\hline
\end{tabular}

sequential reconstruction.

The different types of flaps are listed in Table 2. Eleven patients $(19.0 \%)$ received two free flap procedures, and $10 \mathrm{pa}-$ tients (17.2\%) underwent three or more total operations. Thirty-four patients $(58.6 \%)$ were treated using a sequential free flap reconstruction, while 24 patients $(41.4 \%)$ received local flaps. An anterolateral thigh free flap was used for 11 patients (19.0\%), followed by a radial forearm free flap in 10 patients $(17.2 \%)$ and a rectus abdominis free flap in six patients $(10.3 \%)$. A pectoralis major flap was used for 14 patients (24.1\%), while five (8.6\%) were treated with a temporalis muscle flap.
Table 2. Types of flaps used

\begin{tabular}{|lc|}
\hline Flap & No. (\%) \\
\hline Free flap & $34(58.62)$ \\
Anterolateral thigh & $11(18.97)$ \\
Radial forearm & $10(17.24)$ \\
Rectus abdominis & $6(10.34)$ \\
Vastus lateralis & $3(5.17)$ \\
Latissimus dorsi & $2(3.45)$ \\
Fibula & $2(3.45)$ \\
Local flap & $24(41.38)$ \\
Pectoralis major & $14(24.14)$ \\
Temporalis & $5(8.62)$ \\
Tongue & $2(3.45)$ \\
Palate & $2(3.45)$ \\
Latissimus dorsi & $1(1.72)$ \\
\hline
\end{tabular}

Table 3. Micro-anastomosis recipient vessels $(n=34)$

\begin{tabular}{|lc|}
\hline Vessel & No. (\%) \\
\hline Artery & \\
Superior thyroid & $26(76.47)$ \\
Facial & $4(11.76)$ \\
Lingual & $3(8.82)$ \\
Transverse cervical & $1(2.94)$ \\
Vein & \\
Branch of IJV & $19(55.88)$ \\
Superior thyroid & $6(17.65)$ \\
EJV & $4(11.76)$ \\
Facial & $4(11.76)$ \\
Transverse cervical & $1(2.94)$ \\
\hline IJV, internal jugular vein; EJV, external jugular vein. & \\
\hline
\end{tabular}

In patients who received a free flap, the superficial thyroid artery was most commonly chosen for arterial micro-anastomosis (26 patients; $76.5 \%$ ), and a branch of the internal jugular vein was the most common site for micro-anastomosis of a vein (19 patients; 55.9\%) (Table 3). The facial artery, lingual artery, transverse cervical artery and superior thyroid vein, external jugular vein, facial vein, and transverse cervical vein were also used for micro-anastomoses. All anastomoses but one were conducted in an end-to-end fashion; in one case, the vein was anastomosed in an end-to-end fashion with a radial forearm free flap. In total, 13 complications occurred. In two patients (3.45\%), re-exploration was performed within 1 week of the reconstruction; in another two patients (3.45\%), re-exploration took place after 1 week. Although partial flap loss was noted in two patients (3.45\%), there were no total losses of any flaps. There was also one case of hematoma (1.72\%) and four cases that required minor revisions (6.90\%) (Table 4).

Patients' swallowing and speech abilities were evaluated at an outpatient clinic. Seventeen patients (29.3\%) who underwent reconstruction of the extra-oropharynx region, such as the 


\section{Table 4. Postoperative complications}

\begin{tabular}{|lc|}
\hline Complications & No. (\%) \\
\hline Re-exploration within 1 week & $2(3.45)$ \\
Total re-exploration & $4(6.90)$ \\
Partial flap loss & $2(3.45)$ \\
Hematoma & $1(1.72)$ \\
Minor revision & $4(6.90)$ \\
\hline
\end{tabular}

cheek, ear, and forehead, had undisrupted swallowing and speech and were not examined. In addition, eight patients (13.8\%) died before the examination. Of the rest of the patients, 12 (20.7\%) were able to ingest solid meals, 14 (24.1\%) ate only soft meals, and seven (12.1\%) could not eat anything. With regard to speech, eight patients (13.8\%) had normal speech, 11 (19.0\%) had an impediment, and 14 (24.1\%) were unable to speak.

\section{DISCUSSION}

This study presents a thorough investigation of reconstruction procedures for recurrent head and neck cancer involving $58 \mathrm{pa}-$ tients during a period of 10 years. At our institution, 26 patients required subsequent repetitive reconstructive surgery. Eleven patients received a subsequent free vascularized flap, while 10 patients underwent three or more reconstructive operations. These subsequent reconstructive procedures were more challenging than the initial operation due to scarring from the first operation, fibrosis caused by radiation, and other structural changes that hindered the surgeon in cases with difficult anatomy [10]. Nonetheless, no patient experienced total flap loss. Our experience suggests that sequential reconstructive surgery is worth considering for recurrent head and neck cancer patients, even if they have undergone prior reconstructions, such as local and free vascularized flaps. These findings should aid oncologists in the selection of treatment strategies, including wide surgical removal, when patients experience tumor recurrence.

In our study, 24 patients (41.4\%) underwent reconstruction using a locoregional flap. A pectoralis major flap was mostly preferred ( 14 cases; $24.1 \%)$. Some studies have suggested that in appropriate surgical locations and in cases with contraindications for microvascular surgery, a pectoralis major flap should still be considered for head and neck reconstruction despite the current trend toward microvascular reconstruction [11-13]. Other types of locoregional flaps have also been used, such as a temporalis muscle flap or a palatal flap. However, considering the location and size of the defect following a wide tumor resection, a pectoralis major flap was the most adequate locoregional flap. In another study, the authors suggested that a trapezius island myocutaneous flap was also a suitable option for head and neck reconstruction [14]. However, our surgeons did not use this technique; moreover, most of them preferred free flaps, such as an anterolateral thigh free flap, which was used in 11 cases $(20.0 \%)$, followed by a radial forearm free flap in 10 cases (17.2\%). In general, the length or diameter of the pedicle of an anterolateral thigh flap and a radial forearm flap are known to be adequate for head and neck reconstructions [10,15-17]. In this study, the choice of flap to use in a patient with recurrent cancer was the same as that in a primary cancer case.

Out of 34 free flap cases, the superficial temporal artery was used in 26 cases (76.47\%), and a branch of the internal jugular vein was employed in 19 cases (55.88\%) for micro-anastomoses. Even in patients who underwent repeated reconstruction, no flap loss was noted. In some studies, repeated free flap procedures were successfully performed by re-using the same vessels as the prior operation, and no flap failures occurred $[10,18]$. According to our study, the use of familiar vessels yields positive outcomes, even when structural changes are present due to prior radiation or reconstruction.

In a previous study, several episodes of microvascular reconstruction were associated with postoperative complications, such as a longer hospital stay, total flap failure, fistula, infection, and anastomosis thrombosis [19]. In the current study, postoperative complications were noted in 13 cases (22.4\%). Total reexplorations were performed in four cases $(6.90 \%)$. Of these cases, two $(3.45 \%)$ occurred within 1 week, which may suggest thrombosis or technical factors such as the micro-anastomosis technique used. However, no flap became detached. In every case, appropriate patient-oriented judgment was required to determine the best treatment. The seven patients with impaired swallowing ability and 14 patients with impaired speech ability presented similar results to those of the prior assessment after primary reconstruction.

Our study had several limitations. First, the study was a retrospective review performed at a single institution that was entirely dependent on medical records. Second, since some cases were so recent, the overall long-term survival rate was not available. Patients who experience recurrent head and neck cancer are considered to have a poor prognosis, so further investigations should be carried out in the future. Third, although the surgeons at our institution are sufficiently qualified to perform several reconstructive operations, their choice of flaps was limited. We did not use relatively unfamiliar flaps, such as trapezius island myocutaneous flaps or superficial circumflex iliac artery perforator free flaps.

The oncologic prognosis for recurrent head and neck cancer 
has gradually improved, and patients are expected to live longer than before [20]. As the long-term survival rate increases in these patients, it will be necessary to strongly recommend performing reconstructive surgery to obtain satisfactory results compared to those produced by conservative care [21-23].

\section{NOTES}

\section{Conflict of interest}

No potential conflict of interest relevant to this article was reported.

\section{Ethical approval}

The study was approved by the Institutional Review Board of Severance Hospital (IRB No. 4-2019-0365) and performed in accordance with the principles of the Declaration of Helsinki.

\section{Author contribution}

Conceptualization: Lee WJ. Date curation: Lee WJ. Formal analysis: Chung SW, Byun IH. Methodology: Chung SW, Byun IH, Lee WJ. Project administration: Chung SW, Byun IH, Lee WJ. Visualization: Chung SW, Byun IH. Writing - orginal draft: Chung SW. Writing - review \& editing : Chung SW. Approval of final manuscript: all authors.

\section{ORCID}

Soon Won Chung https://orcid.org/0000-0002-4761-3942

Il Hwan Byun https://orcid.org/0000-0001-7337-3164

Won Jai Lee https://orcid.org/0000-0003-3056-0503

\section{REFERENCES}

1. Cooper JS, Porter K, Mallin K, et al. National Cancer Database report on cancer of the head and neck: 10-year update. Head Neck 2009;31:748-58.

2. Urken ML, Weinberg H, Buchbinder D, et al. Microvascular free flaps in head and neck reconstruction: report of 200 cases and review of complications. Arch Otolaryngol Head Neck Surg 1994;120:633-40.

3. Kroll SS, Evans GR, Goldberg D, et al. A comparison of resource costs for head and neck reconstruction with free and pectoralis major flaps. Plast Reconstr Surg 1997;99:1282-6.

4. Varvares MA, Lin D, Hadlock T, et al. Success of multiple, sequential, free tissue transfers to the head and neck. Laryngoscope 2005; 115:101-4.

5. Vermorken JB, Specenier P. Optimal treatment for recurrent/metastatic head and neck cancer. Ann Oncol 2010;21 Suppl 7:vii252-61.
6. Chen W, Yang Z, Zhang D, et al. Second salvage surgery with extended vertical lower trapezius island myocutaneous flap reconstruction for advanced re-recurrent oral and oropharyngeal squamous cell carcinoma. Int J Oral Maxillofac Surg 2014;43:531-8.

7. Gupta AK, McKenna WG, Weber CN, et al. Local recurrence in head and neck cancer: relationship to radiation resistance and signal transduction. Clin Cancer Res 2002;8: 885-92.

8. Demirkan F, Wei FC, Chen HC, et al. Microsurgical reconstruction in recurrent oral cancer: use of a second free flap in the same patient. Plast Reconstr Surg 1999;103:829-38.

9. Forastiere A, Koch W, Trotti A, et al. Head and neck cancer. N Engl J Med 2001;345:1890-900.

10. Hanasono MM, Barnea Y, Skoracki RJ. Microvascular surgery in the previously operated and irradiated neck. Microsurgery 2009;29:1-7.

11. Avery CM, Crank ST, Neal CP, et al. The use of the pectoralis major flap for advanced and recurrent head and neck malignancy in the medically compromised patient. Oral Oncol 2010;46:829-33.

12. Kekatpure VD, Trivedi NP, Manjula BV, et al. Pectoralis major flap for head and neck reconstruction in era of free flaps. Int J Oral Maxillofac Surg 2012;41:453-7.

13. Avery CM, Gandhi N, Peel D, et al. Indications and outcomes for 100 patients managed with a pectoralis major flap within a UK maxillofacial unit. Int J Oral Maxillofac Surg 2014;43:546-54.

14. Chen WL, Li J, Yang Z, et al. Extended vertical lower trapezius island myocutaneous flap in reconstruction of oral and maxillofacial defects after salvage surgery for recurrent oral carcinoma. J Oral Maxillofac Surg 2007;65:205-11.

15. Ali RS, Bluebond-Langner R, Rodriguez ED, et al. The versatility of the anterolateral thigh flap. Plast Reconstr Surg 2009;124(6 Suppl):e395-407.

16. Wei FC, Jain V, Celik N, et al. Have we found an ideal softtissue flap?: an experience with 672 anterolateral thigh flaps. Plast Reconstr Surg 2002;109:2219-26.

17. Chana JS, Wei FC. A review of the advantages of the anterolateral thigh flap in head and neck reconstruction. Br J Plast Surg 2004;57:603-9.

18. Nakayama B, Kamei Y, Toriyama K, et al. Usefulness of a first transferred free flap vascular pedicle for secondary microvascular reconstruction in the head and neck. Plast Reconstr Surg 2002;109:1246-53.

19. Offodile AC 2nd, Chang KP, Chen HH, et al. Feasibility and outcomes of the third or more episodes of sequential microvascular reconstruction for recurrent or second pri- 
mary oral cancer. Ann Surg Oncol 2016;23:3765-72.

20. Hanasono MM, Corbitt CA, Yu P, et al. Success of sequential free flaps in head and neck reconstruction. J Plast Reconstr Aesthet Surg 2014;67:1186-93.

21. Tsue TT, Desyatnikova SS, Deleyiannis FW, et al. Comparison of cost and function in reconstruction of the posterior oral cavity and oropharynx: free vs pedicled soft tissue transfer. Arch Otolaryngol Head Neck Surg 1997; 123:731-7.
22. Chepeha DB, Annich G, Pynnonen MA, et al. Pectoralis major myocutaneous flap vs revascularized free tissue transfer: complications, gastrostomy tube dependence, and hospitalization. Arch Otolaryngol Head Neck Surg 2004;130: 181-6.

23. Hanasono MM, Friel MT, Klem C, et al. Impact of reconstructive microsurgery in patients with advanced oral cavity cancers. Head Neck 2009;31:1289-96. 